\title{
Both ongoing alpha and visually induced gamma oscillations show reliable diversity in their across-site phase-relations
}

\author{
Freek van Ede, ${ }^{1}$ Stan van Pelt, ${ }^{1}$ Pascal Fries, ${ }^{1,2}$ and Eric Maris ${ }^{1}$ \\ ${ }^{1}$ Donders Institute for Brain, Cognition and Behaviour, Radboud University Nijmegen, Nijmegen, The Netherlands; \\ and ${ }^{2}$ Ernst Strüngmann Institute (ESI) for Neuroscience in Cooperation with Max Planck Society, Frankfurt, Germany
}

Submitted 7 October 2014; accepted in final form 8 December 2014

van Ede F, van Pelt S, Fries P, Maris E. Both ongoing alpha and visually induced gamma oscillations show reliable diversity in their across-site phase-relations. J Neurophysiol 113: 1556-1563, 2015. First published December 10, 2014; doi:10.1152/jn.00788.2014.Neural oscillations have emerged as one of the major electrophysiological phenomena investigated in cognitive and systems neuroscience. These oscillations are typically studied with regard to their amplitude, phase, and/or phase coupling. Here we demonstrate the existence of another property that is intrinsic to neural oscillations but has hitherto remained largely unexplored in cognitive and systems neuroscience. This pertains to the notion that these oscillations show reliable diversity in their phase-relations between neighboring recording sites (phase-relation diversity). In contrast to most previous work, we demonstrate that this diversity is restricted neither to low-frequency oscillations nor to periods outside of sensory stimulation. On the basis of magnetoencephalographic (MEG) recordings in humans, we show that this diversity is prominent not only for ongoing alpha oscillations $(8-12 \mathrm{~Hz})$ but also for gamma oscillations $(50-70 \mathrm{~Hz})$ that are induced by sustained visual stimulation. We further show that this diversity provides a dimension within electrophysiological data that, provided a sufficiently high signal-to-noise ratio, does not covary with changes in amplitude. These observations place phase-relation diversity on the map as a prominent and general property of neural oscillations that, moreover, can be studied with noninvasive methods in healthy human volunteers. This opens important new avenues for investigating how neural oscillations contribute to the neural implementation of cognition and behavior.

gamma oscillations; human; magnetoencephalography; neural oscillations; phase-relation

OVER THE PAST 20 YEARS, neural oscillations have come to the foreground in the scientific study of the neural implementation of cognition and behavior. Indeed, these oscillations have now been implicated in a multitude of neural and cognitive computations (as reviewed in, e.g., Buzsáki and Draguhn 2004; Fries 2005; Hari and Salmelin 1997; Jensen et al. 2007).

To date, most studies have investigated neural oscillations with regard to one or more of the following three properties: amplitude, phase, and/or phase coupling (such as between two distant sources or between field oscillations and action potentials). In this report, we demonstrate the existence of a fourth property that is intrinsic to oscillatory neural activity. This property pertains to the observation that the phase-relations between oscillations recorded at neighboring sites are highly and reliably diverse. We term this property phase-relation diversity.

Address for reprint requests and other correspondence: E. Maris, Radboud Univ. Nijmegen, Donders Institute for Brain, Cognition and Behaviour, Centre for Cognition, Montessorilaan 3, 6525 HR Nijmegen, The Netherlands (e-mail: e.maris@donders.ru.nl).
Several previous studied have already alluded to the notion of phase-relation diversity in the context of "traveling waves," and recent studies have pointed to important computational roles for such diversity (Agarwal et al. 2014; Lubenov and Siapas 2009). However, to date, the demonstration of such diversity has been largely restricted to low-frequency oscillations that are prominent in the absence of sensory processing (e.g., Agarwal et al. 2014; Bahramisharif et al. 2013; Hindriks et al. 2014; Hughes 1995; Nunez 2000; Lubenov and Siapas 2009; van der Meij et al. 2012). In fact, in a prominent review article on the subject, a central claim is that this type of diversity is "typically present during periods outside of stimulation, while synchronous activity dominates in the presence of a strong stimulus" (Ermentrout and Kleinfeld 2001). Importantly, however, if such diversity plays an important role in neural computations, it should also be prominent for neural activity that prevails during periods of active stimulus processing.

We recently demonstrated that high-frequency gamma oscillations during sustained visual stimulation also exhibit reliable diversity in their phase-relations between nearby recording sites in monkey V4 (Maris et al. 2013). Here we investigated whether this generalizes to humans and to extracranial recordings. On the basis of magnetoencephalographic (MEG) recordings, we confirm in humans that phase-relation diversity is a general property of neural oscillations that is restricted neither to the lower frequencies nor to periods outside of stimulation. In particular we show that, like ongoing alpha oscillations $(8-12 \mathrm{~Hz})$, gamma oscillations $(50-70 \mathrm{~Hz})$ that are induced by sustained visual stimulation also exhibit prominent phase-relation diversity.

\section{MATERIALS AND METHODS}

Participants. We analyzed data from two experiments. Experiment 1 focused on ongoing alpha oscillations. Eight healthy participants (6 men, 2 women; age range: $24-50 \mathrm{yr}$ ) participated in this experiment. One participant was excluded from the analysis because he showed no clear alpha oscillations in the eyes-open condition. Experiment 2 focused on visually induced gamma oscillations. This experiment was originally conducted to study genetic contributions to these oscillations. In the original experiment, 160 healthy participants participated. For our purpose, we first focused on data from the 10 participants ( 2 men, 8 women; age range: $18-28 \mathrm{yr}$ ) who showed the strongest gamma response to visual stimulation. We used this selection because reliable phase-relation estimates require sufficient spectral energy. Thus zooming in on those participants with the highest amplitudes ensures an optimal signal-to-noise ratio (SNR) for investigating phase-relations. Critically, however, besides facilitating SNR, this amplitude-based selection is independent of the critical aspect of our study, which concerns the diversity of phase-relations. For control 
purposes, we also replicated our main results in another sample of 20 participants (7 men, 13 women; age range: 18-34 yr) whose gamma responses to visual stimulation were in the intermediate range. Both experiments were conducted according to protocols reviewed and approved by the local ethics committee (Committee on Research Involving Human Subjects, Region Arnhem-Nijmegen, The Netherlands).

Experiment 1. Experiment 1 consisted of two parts. In part 1, every 8,10 , or $12 \mathrm{~s}$, participants heard a man's voice instructing them in alternation to either open or close their eyes. This continued for 30 min. Part 1 was immediately followed by part 2 , consisting of two continuous blocks of $5 \mathrm{~min}$ : one with eyes open and the other with eyes closed. Data were cut into epochs of $2 \mathrm{~s}$. Epochs of parts 1 and 2 were analyzed together. We analyzed $\sim 500$ epochs per condition.

Experiment 2. Participants performed a visual change detection task with inward-moving concentric gratings (similar to Hoogenboom et al. 2006; van Pelt et al. 2012). Gratings had a diameter of $7.1^{\circ}$ visual angle and were presented foveally. Participants were instructed to respond as fast as possible to a speed change of the inward motion. Responses were made with the right index finger. The speed change occurred at an unpredictable time point between 750 and 3,000 ms after stimulus onset. We cut the data into epochs of $750 \mathrm{~ms}$ and only used epochs prior to the speed change. In different trials, stimuli were presented at either of three different speeds of the inward motion $(0$, $0.33 \%$, and $0.66 \%$ s) and at two different contrast levels $(50 \%$ and $100 \%$ contrast). Trial types were randomly intermixed. We only analyzed trials in which the speed of the inward motion was $0.66 \%$ s. Because the spatial frequency of the grating was 3 cycles $/{ }^{\circ}$, the frequency of black/white transitions (which were sinusoidal) at a given position in the visual field was $\sim 4.5 \mathrm{~Hz}$, which is well below the frequency of the induced gamma oscillations $(\sim 60 \mathrm{~Hz})$. The task lasted $1 \mathrm{~h}$. We analyzed $\sim 250$ epochs per condition.

$M E G$ recordings and preprocessing. In both experiments, data were collected with a CTF MEG system (Port Coquitlam, BC, Canada) containing 275 axial gradiometers (referred to as MEG sites). Data were high-pass filtered at $300 \mathrm{~Hz}$, sampled at $1,200 \mathrm{~Hz}$, and stored for off-line analyses. Data were analyzed in MATLAB, using FieldTrip (Oostenveld et al. 2011) in combination with custom code. Fifty-hertz line noise was removed with a filter based on the discrete Fourier transform. Epochs contaminated by artifacts were removed after visual inspection. From the data of experiment 1, we additionally subtracted two heartbeat components with independent component analysis (ICA; Bell and Sejnowski 1995). We subtracted those components whose time courses were highly coherent with the electrocardiogram (ECG). In experiment 2 we had not recorded the ECG, and so we did not remove these components.

Analysis of phase-relations. We performed spectral analysis, using a multitaper approach to achieve optimal spectral concentration (Mitra and Pesaran 1999; Percival and Walden 1993). We applied tapers from a family of orthogonal prolate spheroidal functions. For all analyses on alpha oscillations (experiment 1) we applied $\pm 2 \mathrm{~Hz}$ smoothing; for gamma oscillations (experiment 2) we applied $\pm 5 \mathrm{~Hz}$ smoothing.
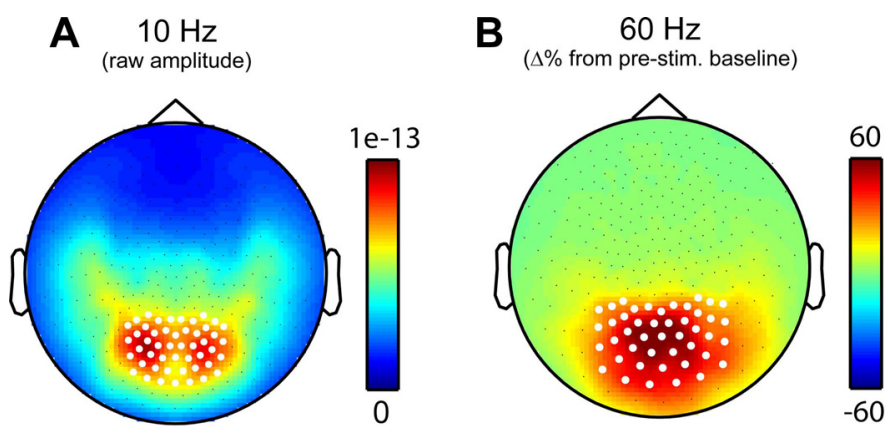

In multitaper estimation, for each taper the data segment is multiplied with that taper and then Fourier transformed, giving the windowed Fourier transform $\tilde{\mathrm{x}}_{\mathrm{k}}(f)$ :

$$
\tilde{\mathrm{x}}_{\mathrm{k}}(f)=\sum_{t=1}^{N} w_{k}(t) x_{t} e^{-2 \pi i f t}
$$

where $x_{t}(t=1,2, \ldots N)$ is the time series of the signal under consideration and $w_{k}(t)(k=1,2, \ldots K)$ are $K$ orthogonal taper functions. The multitaper estimates for the spectrum $S_{x x}(f)$ and the cross-spectrum $S_{x y}(f)$ are given by

$$
\begin{aligned}
& S_{x x}(f)=\frac{1}{K} \sum_{k=1}^{K}\left|\tilde{x}_{k}(f)\right|^{2} \\
& S_{x y}(f)=\frac{1}{K} \sum_{k=1}^{K} \tilde{x}_{k}(f) \tilde{y}_{k}^{*}(f)
\end{aligned}
$$

For the analysis of phase-relations, we used the phase angle of the cross-spectrum, which gives the preferred (i.e., average) phase-relation between the two time series, $x_{t}$ and $y_{t}$ (i.e., between the two sites of a given site pair), and ranges between $[-\pi,+\pi]$.

We calculated phase-relations for all site pairs within a cluster of selected sites. Site clusters were selected based on the average amplitude topography across all participants and conditions in the experiment, as depicted in Fig. 1. We used 51 sites for experiment 1 and 47 sites for experiment 2. Critically, this selection is based on amplitude and is therefore independent of the critical aspect of our study, which concerns phase-relation diversity. As a control, we also calculated systematic phase-relation diversity spectra on the basis of site clusters that were not data driven. In separate analyses, we used all sites posterior to the midline $(n=128)$ as well as all MEG sites $(n=275)$.

Analysis of systematic phase-relation diversity. We quantified the degree to which phase-relations were both reliable and diverse across all selected site pairs. Our approach is schematically depicted in Fig. 2 and described in detail below.

To quantify systematic phase-relation diversity, we build on a previously developed metric termed over-site-pair phase-relation diversity (SPHARED; Maris et al. 2013). Although we used slightly modified calculations (to deal with dipolar patterns), we also refer here to our metric as SPHARED.

SPHARED is based on a split-half procedure to separate systematic from unsystematic phase-relations. Per condition, we divided all epochs into two sets: odd-numbered epochs and even-numbered epochs. For both sets, we calculated epoch-averaged phase-relations between all selected site pairs. The rationale behind this is that if a site pair has a reliable phase-relation, then this relation should be observed in both sets of epochs. In contrast, if the phase-relation is not reliable, then both sets will produce a different phase-relation.

The pairs of phase-relations (with one element from the oddnumbered and the other from the even-numbered epochs) are points in a space that is defined by two circular axes, one for the odd and the other for the even epochs. Although a circular axis ranges from $-\pi$ to $+\pi$, for simplicity we first explain SPHARED by restricting

Fig. 1. Site selections based on the grand average amplitude topographies. A: site selection for alpha oscillations in experiment $1(n=51)$. B: site selection for gamma oscillations in experiment $2(n=47)$. Sites involve axial gradiometers. 
Fig. 2. Schematic of analysis of systematic phase-relation diversity. See Analysis of systematic phase-relation diversity for details.

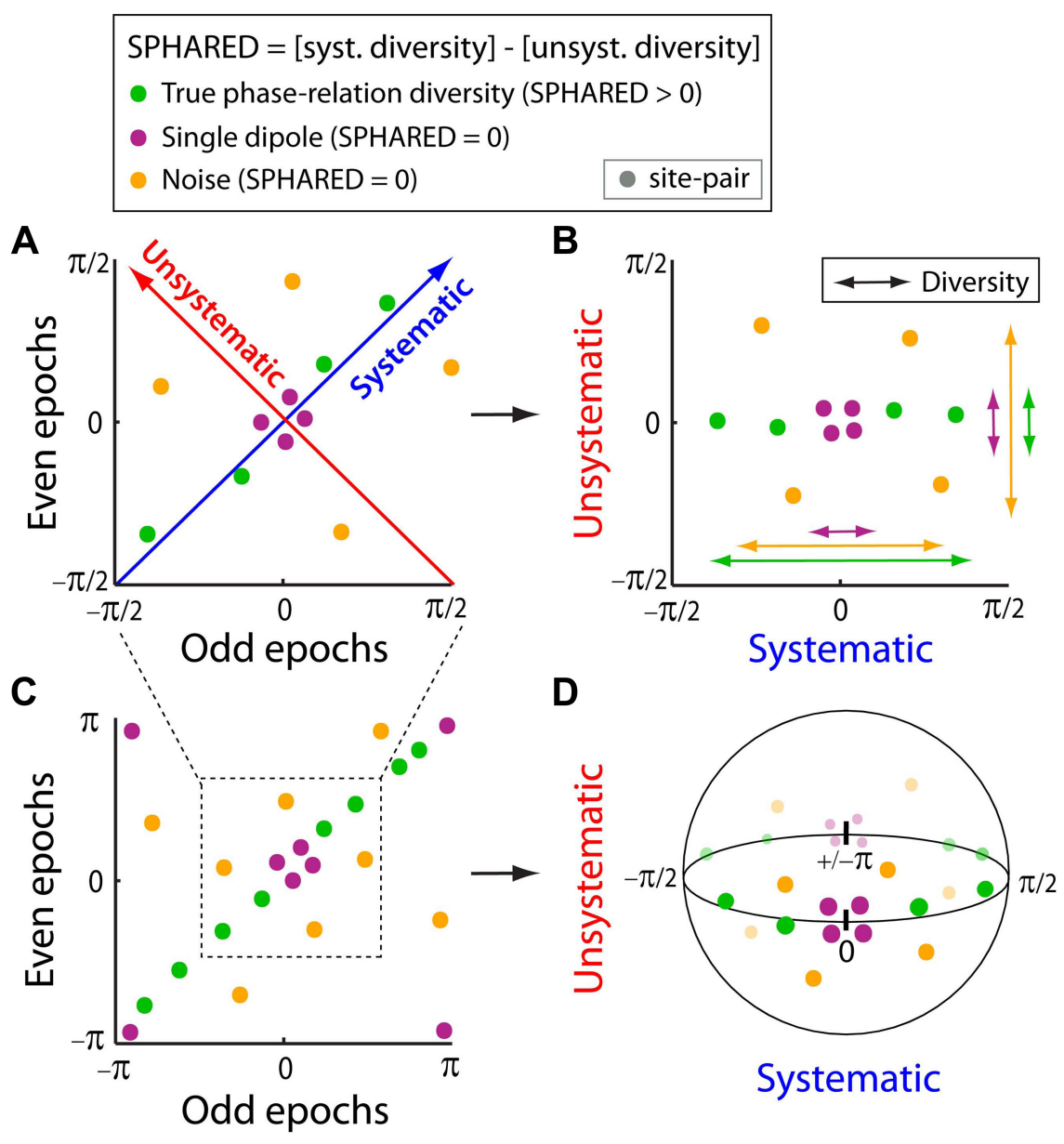

ourselves to all systematic phase-relations in the range $[-\pi / 2,+\pi / 2]$ (Fig. 2, $A$ and $B$ ). We first project every point (site pair) onto two new axes, with one axis corresponding to the systematic component in the phase-relations and the other to the unsystematic component (Fig. $2 B)$. Per site pair, its location on the systematic axis is defined by the average phase-relation between the odd and even epochs (represented as points on the unit circle in the complex plane) and the projection on the unsystematic axis is defined as the difference in the phaserelations between the odd and even epochs. Because noise is twice as small on the systematic projection (because noise is reduced by averaging but not by subtracting), we divided all values on our unsystematic axis by 2 .

SPHARED is based on the following rationale. If there is diversity in the phase-relations between all site pairs and if this is reliable, then the diversity across the systematic axis (which contains both systematic diversity and noise) should be larger than the diversity across the unsystematic axis (which contains only noise). SPHARED is therefore calculated as the difference in diversity between the systematic and the unsystematic axes (systematic minus unsystematic). Diversity is quantified as the absolute deviation from phase-relations of 0 , averaged across all site pairs. Thus

$$
\operatorname{SPHARED}(f)=\frac{1}{S} \sum_{s=1}^{S}\left(\left|a_{s}(f)\right|-\left|b_{s}(f)\right|\right)
$$

Where $a_{s}$ and $b_{s}(s=1,2, \ldots, S)$ represent the epoch-averaged phase angles of $S$ site pairs on the systematic and unsystematic projections, respectively. SPHARED is zero when 1) there is no diversity in the phase-relations across site pairs (the dipole scenario in Fig. 2, see Fig. $2 B$ ) or 2) when this diversity is not reliable (the noise scenario in Fig. 2). In fact, SPHARED will only be positive when there are nonzero phase-relations and when these are reliable (the true phase-relation diversity scenario in Fig. 2). We verified this with simulated data. Note that in Maris et al. (2013), where SPHARED was introduced, it was based on the difference between two circular means between the systematic and the unsystematic projections (rather than the absolute deviations from phase angles of 0 between both projections). However, this slight difference in calculation does not affect its interpretation.

We now consider systematic phase-relations across the full circle (i.e., ranging between $[-\pi,+\pi]$ ). The full geometrical representation of the odd and even epoch phase-relations is the surface of a sphere (Fig. 2D). So far, we have only considered half of this sphere (see the dashed lines in Fig. 2C). Looking at the phase-relations across the full circle, we now note that a single current dipole also produces phaserelation diversity, simply because its two poles differ by $\pi$. Specifically, in the MEG signal a single current dipole will produce a positive (outward) flux in recording sites on one side of the dipole and a negative (inward) flux in sites on the opposite side of the dipole. Therefore, at the level of the site pairs, an oscillating dipole will produce phase-relations of both 0 and $\pi$, depending on whether the two sites of a pair are located in the same or opposite poles. We are not interested in this type of diversity because it is produced by a single underlying source. To deal with this, we split our site pair data into two halves: one half with systematic phase-relations in the range $[-\pi / 2,+\pi / 2]$ (thus centered at 0 ) and one half with phase-relations outside this range (thus centered at $\pm \pi$ ). In other words, we split our data according to the two halves of the sphere (Fig. 2D). We calculated SPHARED separately for each half of the sphere and subsequently pooled these values. Computationally, this was achieved by separating all site pairs with systematic components outside the 
range $[-\pi / 2,+\pi / 2]$ and applying a phase shift of $\pi$ to these phase-relations before calculating SPHARED. As evident from Fig. $2 D$, single dipoles only produce diversity between both halves of the sphere, not within either of them. Therefore, our two-step SPHARED metric is insensitive to all diversity that may result from single dipoles.

\section{RESULTS}

We investigated the distribution of phase-relations across MEG sites for ongoing alpha oscillations $(8-12 \mathrm{~Hz})$ as well as for visually induced gamma oscillations $(50-70 \mathrm{~Hz})$.

We first describe the main phenomenon (phase-relation diversity) as well as our analysis approach for ongoing alpha oscillations (Fig. 3, $A-D$ ). Figure $3 A$ depicts the topography of alpha amplitude for a representative participant during a resting-state measurement with the eyes closed. To evaluate phaserelations, we first selected the recording site with the highest amplitude as a reference site and plotted the epoch-averaged phase-relations between this and all other sites. This is depicted in Fig. 3B. If alpha oscillations originated from a single oscillating dipole, then all between-site phase-relations would be either 0 or $\pi$ (depending on whether the sites are located in the same or the opposite magnetic poles). Instead, we observe a rich palette of phase-relations (i.e., phase-relation diversity). This implies multiple underlying sources (ranging from 2 to a continuum, see DISCUSSION) that oscillate at different phases.

To evaluate the reliability of these phase-relations, we made use of a split-half procedure (see also MATERIALS AND METHODS). For this, we zoomed in on a fixed cluster of sites at which alpha amplitude was most pronounced (see Fig. $1 A)$. For all site pairs in this cluster, we calculated the average phase-relations in two independent splits of the data: odd and even epochs. Figure $3 C$ shows the outcomes of this approach for three representative frequencies, where individual data points represent site pairs. Specifically at 10 $\mathrm{Hz}$ (and not at 5 and $40 \mathrm{~Hz}$ ), we observe phase-relations that are both diverse (spread out over the plotted range) as well as reliable (highly similar between the odd and the even epochs). To quantify this pattern of interest, we derived a metric of systematic phase-relation diversity (MATERIALS AND METHODS; see also Maris et al. 2013). Figure 3D shows how this metric varies as a function of frequency for this partic-

\section{Ongoing alpha oscillations}
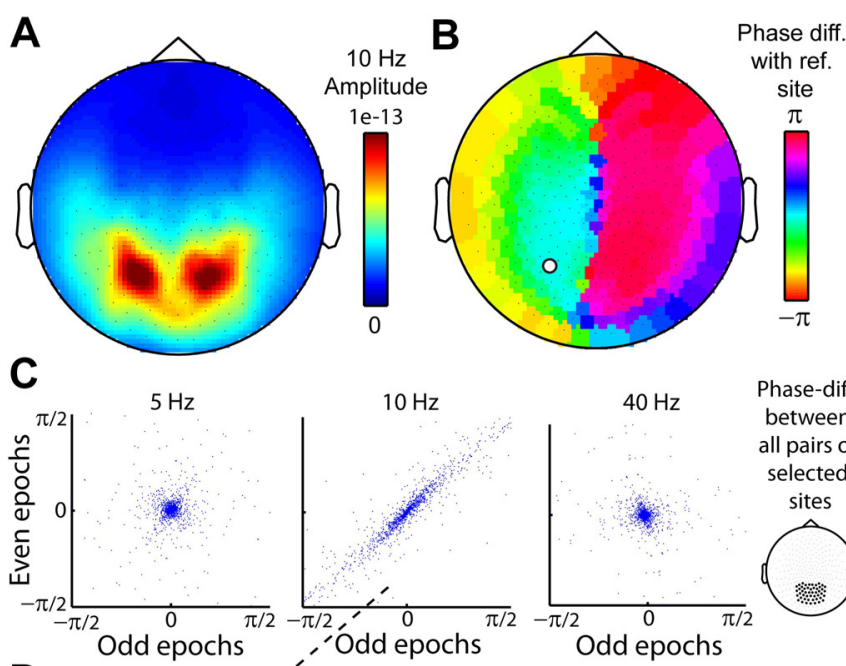

Phase-diff between all pairs of selected sites

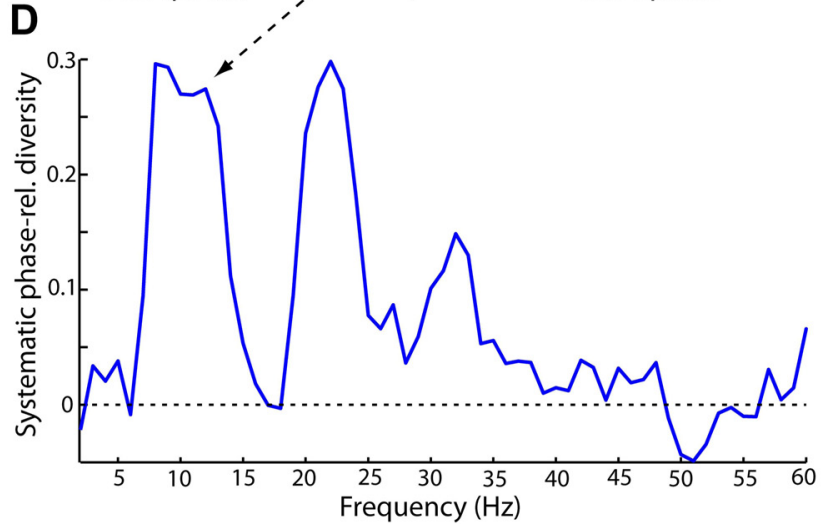

Visually-induced gamma oscillations
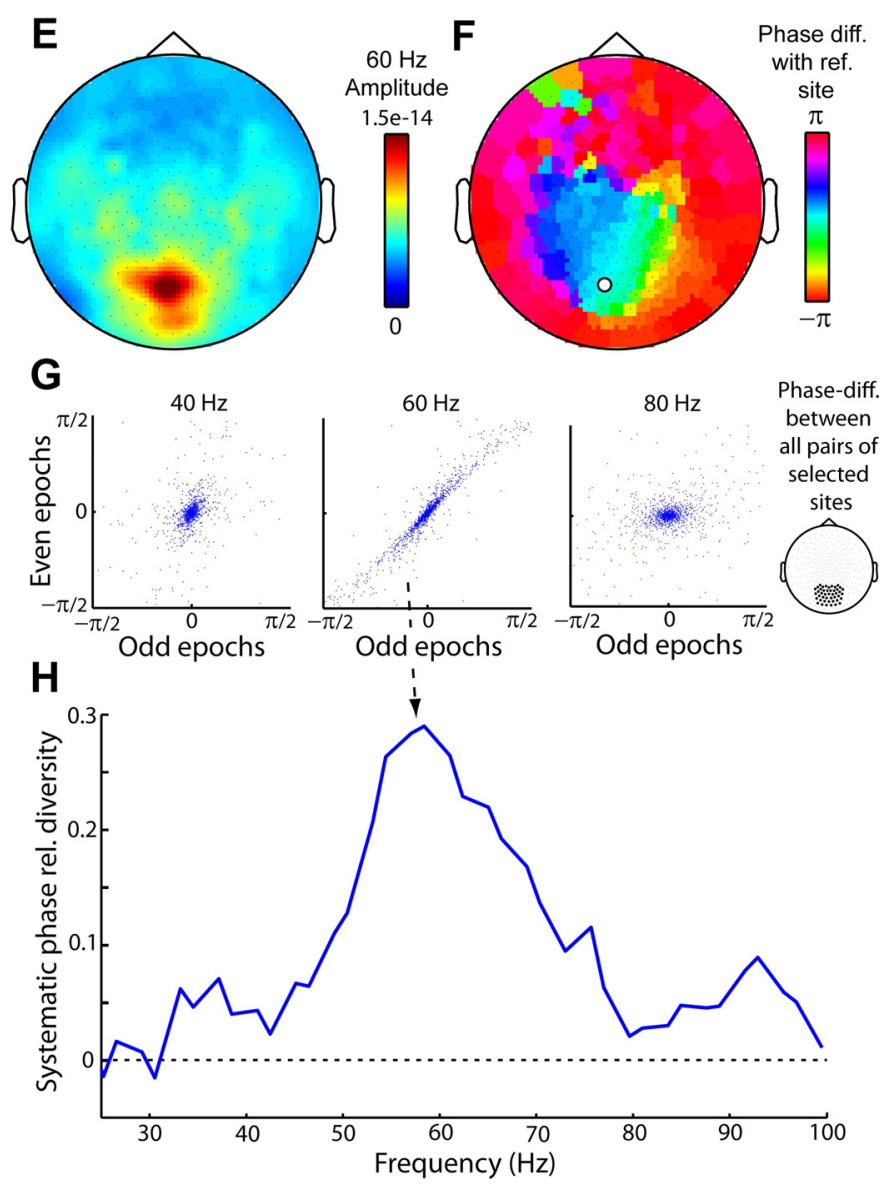

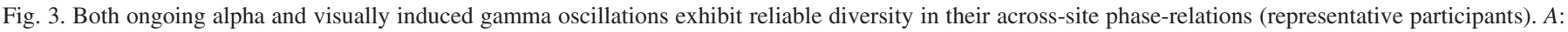

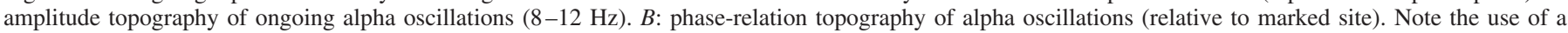

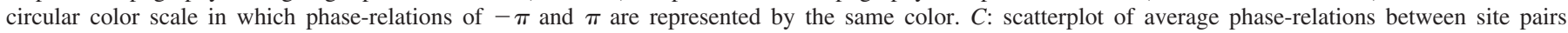

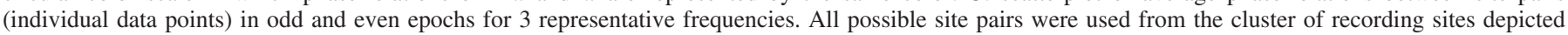

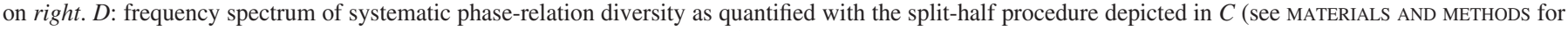

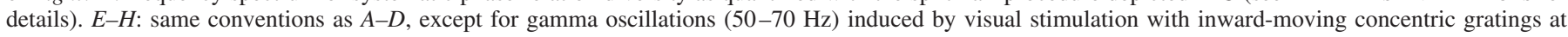
full contrast. Grand average spectra are depicted in Fig. 4, $C$ and $D$. 
ular participant and shows clear peaks at the alpha (around $10 \mathrm{~Hz}$ ) and beta (around $20 \mathrm{~Hz}$ ) frequency bands.

If the observed phase-relation diversity is a general property of neural oscillations, it should also be manifest for other types of oscillations. To assess this, we also analyzed gamma oscillations that are induced by sustained visual stimulation (for stimulation we used inward-moving concentric gratings similar to Hoogenboom et al. 2006 and van Pelt et al. 2012). As depicted in Fig. 3, $E-H$, visually induced gamma oscillations (around $60 \mathrm{~Hz}$ ) also show reliable diversity in their phaserelations across MEG sites.

We next assessed the extent to which the observed phaserelation diversity is replicable across participants and experimental conditions. Figure 4 depicts the grand average amplitude (Fig. 4, $A$ and $B$ ) and systematic phase-relation diversity (Fig. 4, $C$ and $D$ ) spectra for the different experimental conditions in the two experiments. Two key points stand out. First, systematic phase-relation diversity is highly replicable across participants. This is the case both for alpha oscillations in the eyes-open and eyes-closed conditions in experiment 1 (Fig. $4 C$ ) and for gamma oscillations during visual stimulation at full and half contrast in experiment 2 (Fig. 4D). In fact, the horizontal lines in Fig. 4, $C$ and $D$, mark where the diversity is significantly different from 0 (no systematic diversity) at the group level, with a $P$ value $<0.001$. Second, this diversity occurs specifically for those frequency bands that show an oscillatory component (as derived from the amplitude spectra). This is also highlighted by the lack of a clear peak in both prestimulus baseline spectra in Fig. $4, B$ and $D$.

Because oscillations differed markedly in amplitude between the experimental conditions (compare blue and red spectra in Fig. 4, $A$ and $B$ ), we were also able to assess whether phase-relation diversity varies with amplitude. This was not the case. As evident from Fig. 4, $C$ and $D$, phase-relation diversity spectra for both alpha and gamma oscillations were highly similar in those conditions that differed markedly in amplitude (again compare blue and red spectra).

Having described our primary findings, we now discuss the outcomes of four control analyses (Fig. 5). First, while Fig. 4 demonstrated that the spectra of phase-relation diversity are highly similar between conditions that differ in amplitude, it may still be that the precise spatial patterns of phase-relations are different. However, as depicted in Fig. 5A, these phaserelation topographies also were highly similar (although we only depict this for the representative participants from Fig. 3, this was the case across our participant samples).

Second, it is possible that our main results are contingent on the fact that our site selection was based on amplitude. However, this turned out not to be the case. Figure $5 B$ shows that when all sites posterior to the midline $(n=128)$ or even all MEG sites $(n=275)$ were used, qualitatively similar results were obtained (i.e., phase-relation diversity spectra still peaked at alpha and gamma frequencies and were still highly similar between conditions). We did note for gamma oscillations that phase-relation diversity values were reduced by including more sites, whereas this was not the case for alpha oscillations. This is likely due to the fact that alpha oscillations are more widespread than gamma oscillations (at least in our recording setup; see Hindriks et al. 2014), as is also suggested by their phase-relation topographies (Fig. $5 A$ ). Possibly this is due to the fact that alpha oscillations have a higher SNR, such that even in distant sites their phases can be estimated reliably.

Third, we observed that phase-relation diversity is highly similar between conditions that differ in amplitude. Does this generalize to amplitude fluctuations within conditions? To address this, we split epochs within each condition into low (second quartile)- and high (third quartile)-amplitude bins (Fig. $5 C$, left; note that we avoided the first and fourth quartiles to minimize the contribution of epochs with no detectable oscil-
Fig. 4. Phase-relation diversity is replicable across participants and does not covary with amplitude. $A$ and $B$ : grand average amplitude spectra across the selected sites in the different experimental conditions. Amplitudes are normalized to $10-\mathrm{Hz}$ amplitude in the eyesopen condition (experiment $1, A$ ) and $60-\mathrm{Hz}$ amplitude in the prestimulus baseline condition (experiment 2, B). $C$ and $D$ : corresponding spectra of systematic phaserelation diversity. Shadings indicate $\pm 1 \mathrm{SE}$ across participants. Horizontal lines mark where the diversity is significantly different from 0 (no systematic diversity), with a $P$ value $<0.001$.
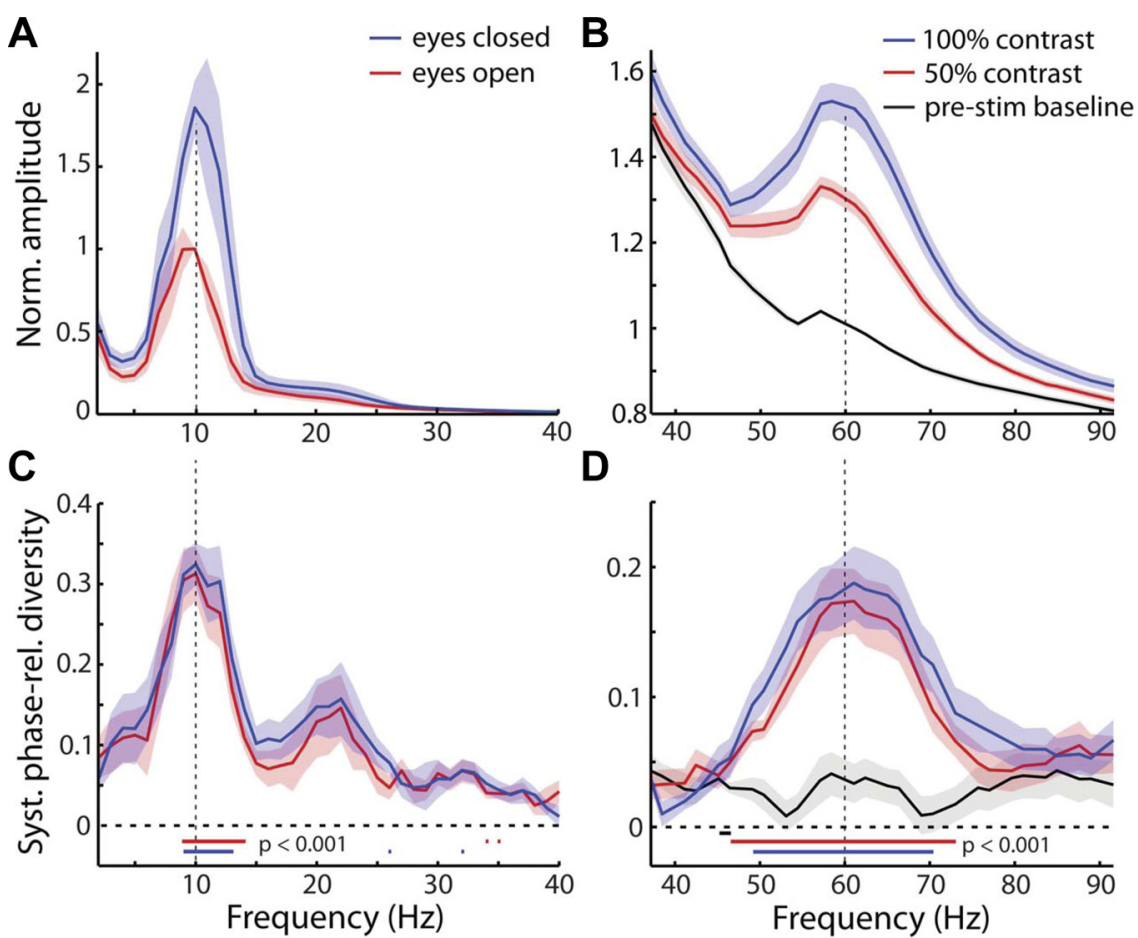
A
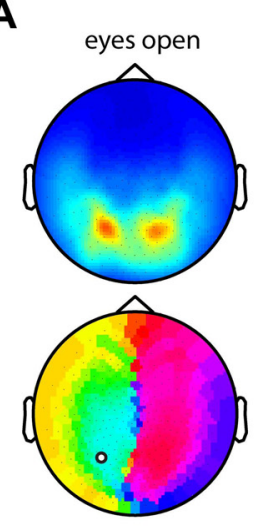
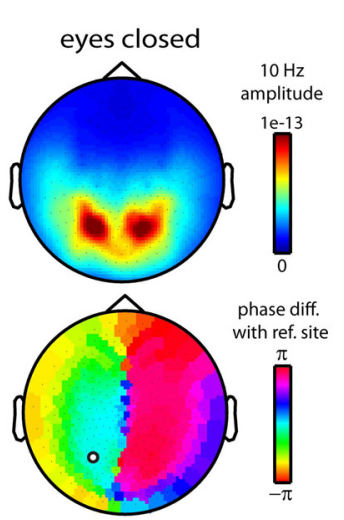
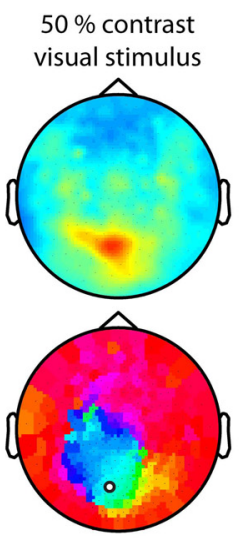

$100 \%$ contrast visual stimulus
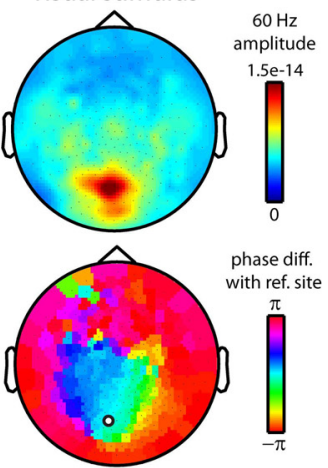

B
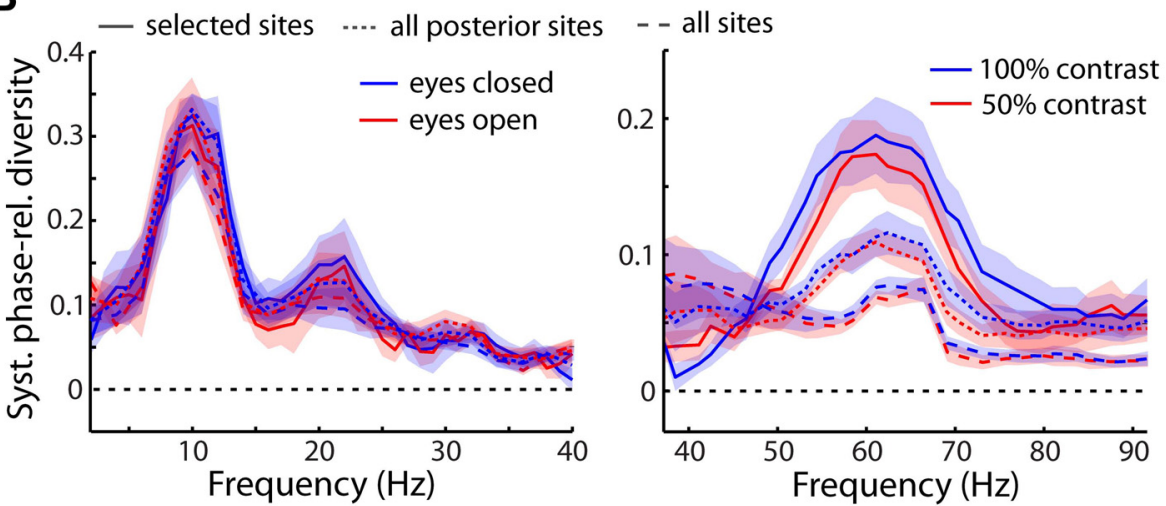

C
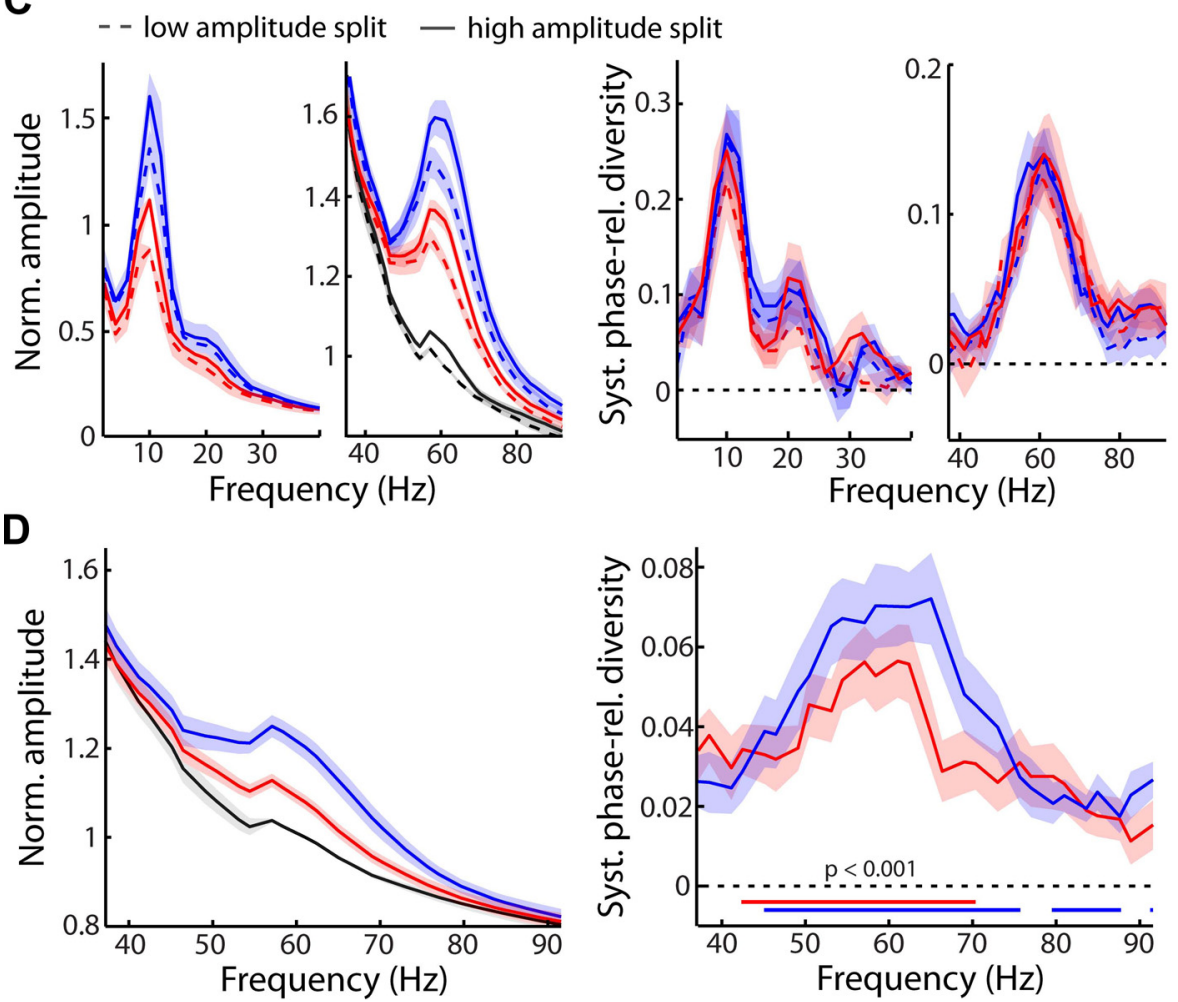

lations or with excessive spectral amplitude that may be caused by artifacts). Again, phase-relation diversity spectra remained remarkably similar, despite differences in amplitude (Fig. 5C, right).
Finally, in our initial analysis for gamma oscillations, we focused on the 10 participants (out of a population of 160) who showed the strongest gamma amplitude increase to visual stimulation. This was done to ensure the highest possible SNR,
Fig. 5. Control analyses. A: topographies of alpha and gamma amplitude and phase-relations for the different experimental conditions. Conventions as in Fig. 3, $A$ and $E$. $B$ : systematic phase-relation diversity spectra based on the selected sites (see Fig. 1), all posterior sites, and all sites. $C$ : amplitude and phase-relation diversity spectra for condition-specific low (2nd quartile)- and high (3rd quartile)-amplitude splits. $D$ : amplitude and systematic phase-relation diversity spectra for another sample of 20 participants who showed intermediate gamma responses to visual stimulation. Horizontal lines mark where the systematic phase-relation diversity is significantly different from 0 (no systematic diversity), with a $P$ value $<0.001$. All depicted data below $40 \mathrm{~Hz}$ are from experiment 1 , and data above $40 \mathrm{~Hz}$ are from experiment 2 . 
which allowed us to estimate phase-relations most reliably. Although this selection is independent of the property of interest (the diversity in these phase-relations), a valid question remains of whether phase-relation diversity of gamma oscillations is also prominent in human MEG recordings from a more representative participant sample. To this end, we drew a second sample of 20 participants whose visual gamma responses were in the intermediate range (ranked between 70 and 89 out of 160). Indeed, these participants showed substantially lower gamma amplitudes than participants in our previous sample (compare Fig. 5D, left, with Fig. 4B). Critically, however, in this sample we also observed highly significant systematic phase-relation diversity that peaked around $60 \mathrm{~Hz}$ (Fig. $5 D, r i g h t)$. Although this diversity was slightly lower for $50 \%$ compared with $100 \%$ contrast visual stimulation, this is likely due to the fact that phase-relation estimates are more susceptible to noise in the $50 \%$ contrast condition, which was characterized by relatively low gamma amplitude in this sample (see Fig. 5D, left).

\section{DISCUSSION}

The main advance provided by this work is the demonstration that diversity in the phase-relations between neighboring recording sites is a prominent and general property of neural oscillations that can, moreover, be studied in humans with noninvasive methods like MEG. This is important because this property has hitherto remained largely unexplored (at least with regard to task-induced oscillations), despite the fact that it may 1) be present in many electrophysiological data sets and 2) serve an important computational role (Agarwal et al. 2014; Lubenov and Siapas 2009; Maris et al. 2013; van der Meij et al. 2012), as we return to below.

To date, phase-relation diversity has been demonstrated almost exclusively for low-frequency theta (Agarwal et al. 2014; Lubenov and Siapas 2009; van der Meij et al. 2012) and alpha (Bahramisharif et al. 2013; Hindriks et al. 2014; Hughes 1995; Nunez 2000) oscillations that occur in the absence of (controlled) stimulation. Here we show that this diversity is also prominent for high-frequency gamma oscillations during sustained visual stimulation (see also Maris et al. 2013). This diversity is therefore restricted neither to low-frequency oscillations nor to periods outside of stimulation. This is important because it suggests that this is a general property of neural oscillations, whose possible functional role therefore also extends to the realm of active stimulus processing. In addition, this work provides two further insights. First, many previous demonstrations of phase-relation diversity have been based on invasive intracranial grid and wire recordings. The present work shows that this diversity is also prominent in extracranial recordings (see also Hindriks et al. 2014; Hughes 1995; Klimesch et al. 2007; Nunez 2000; Nunez and Srinivasan 2005). This is important because it opens the possibility of further studying the functional relevance of this diversity with noninvasive methods in healthy human participants. Second, we show that this diversity provides a dimension within electrophysiological data that does not covary with changes in oscillatory amplitude (given the presence of an oscillatory component and a sufficiently high SNR, as we return to below). Accordingly, the further investigation of phase-relation diversity may result in novel insights into how oscillations contrib- ute to cognition and behavior, over and above insights derived from changes in amplitude and/or coupling strength alone.

Most previous studies demonstrating phase-relation diversity have attributed this to "traveling waves" in which oscillatory phase varies systematically across space in the neural tissue (Ermentrout and Kleinfeld 2001). The diversity reported here may also constitute traveling neural activity. However, because MEG recording sites do not follow the curvature of the underlying tissue, this is difficult to assess at this level of investigation. In fact, modeling work has revealed that diverse phase-relations across distant EEG/MEG sites may reflect intracortical propagation of relatively localized oscillations (Hindriks et al. 2014). As an alternative to traveling waves, the observed diversity may also reflect distinct underlying sources (such as the visual areas V1, V2, V4, MT, etc.) that are phase coupled with distinct latencies (with a minimum of 2 sources whose magnetic fields mix to produce diversity at the sensor level). Indeed, propagation of activity across visual brain areas has been demonstrated previously in MEG (Cottereau et al. 2011) and may also contribute to the diversity observed here. A recent study in monkeys even points to the possibility that both scenarios take place in concert, whereby activity travels within different visual areas while maintaining stable phase delays between visual areas (Muller et al. 2014).

What computational role may phase-relation diversity serve? We recently showed for gamma oscillations in monkey V4 that this diversity changes systematically with visual stimulation as well as covert attention (Maris et al. 2013). Moreover, Agarwal et al. (2014) recently showed for theta oscillations in rat hippocampus that patterns of phase-relations encode the rat's position, and Kösem et al. (2014) showed for entrained oscillations at $1 \mathrm{~Hz}$ that phase shifts (in their case relative to the input stream) can optimize the timing of perceptual processing. These observations suggest a functional role of diverse phaserelations and raise the question of how such diversity might shape neural computations. One possibility is that it complements mechanisms (as outlined in Fries 2005) via which coherent neural oscillations allow selective routing of information between populations. In fact, within a larger population that oscillates coherently, different subpopulations could be segregated on the basis of their phase-relations (Lubenov and Siapas 2009; Maris et al. 2013; van der Meij et al. 2012).

To further assess the functional relevance and computational role of this diversity, it will be critical for future work to address whether and how this diversity changes with task, behavioral, and cohort variables (such as attention, stimulus detection, aging, disease, etc.), as well as how this diversity influences action potential timing and thereby shapes the routing of information within neural circuits. To this end, it would be particularly interesting to demonstrate functional relevance of changes in phase-relation patterns in situations in which amplitude and coupling strength between sources remain unaltered. Interestingly, these aspects could even be investigated in existing data sets for which previous analyses have been blind to the property of phase-relation diversity.

While we have shown that phase-relation diversity can be unaltered with changes in amplitude (Fig. 4), it is important to note that this will only be the case when the SNR allows average phase-relation estimates to be accurately recovered. For the data in Fig. 4, $C$ and $D$, this was likely the case for both the high- and low-amplitude conditions and, as a consequence, 
we obtained comparable systematic phase-relation diversity values. However, we also noted that systematic phase-relation diversity was substantially reduced in a second participant sample whose gamma amplitudes were only moderate (compare Fig. 4, $B$ and $D$, with Fig. $5 D$ ). In this participant sample, it is conceivable that lower SNR resulted in less accurate recovery of the average phase-relations in the two data divisions (odd and even epochs). As a consequence, estimated average phase-relations in the odd and even epochs may have diverged, resulting in an increase in the diversity in the unsystematic projection, and thereby reducing systematic phaserelation diversity values (see the calculation of our measure).

In conclusion, the present work demonstrates that diversity in the across-site phase-relations is a general property of neural oscillations. This property is manifest also for gamma oscillations during stimulus processing and is prominent even in extracranial MEG recordings. These observations open important new avenues for investigating how neural oscillations contribute to the neural implementation of cognition and behavior.

\section{GRANTS}

This work was supported by the Smart Mix Programme of the Netherlands Ministry of Economic Affairs and the Netherlands Ministry of Education, Culture and Science (BrainGain to E. Maris and P. Fries), the European Science Foundation European Young Investigator Award Program (P. Fries), the European Union (HEALTH-F2-2008-200728 to P. Fries), and the LOEWE program ("Neuronale Koordination Forschungsschwerpunkt Frankfurt" to P. Fries).

\section{DISCLOSURES}

No conflicts of interest, financial or otherwise, are declared by the author(s).

\section{AUTHOR CONTRIBUTIONS}

Author contributions: F.v.E., S.V.P., P.F., and E.M. conception and design of research; F.v.E. and S.V.P. performed experiments; F.v.E. and E.M. analyzed data; F.v.E. and E.M. interpreted results of experiments; F.v.E. prepared figures; F.v.E. and E.M. drafted manuscript; F.v.E., S.V.P., P.F., and E.M. edited and revised manuscript; F.v.E., S.V.P., P.F., and E.M. approved final version of manuscript.

\section{REFERENCES}

Agarwal G, Stevenson IH, Berényi A, Mizuseki K, Buzsáki G, Sommer FT. Spatially distributed local fields in the hippocampus encode rat position. Science 344: 626-630, 2014.

Bahramisharif A, van Gerven MA, Aarnoutse EJ, Mercier MR, Schwartz TH, Foxe JJ, Ramsey NF, Jensen O. Propagating neocortical gamma bursts are coordinated by traveling alpha waves. $J$ Neurosci 33: 1884918854, 2013.

Bell AJ, Sejnowski TJ. An information-maximization approach to blind separation and blind deconvolution. Neural Comput 7: 1129-1159, 1995.

Buzsáki G, Draguhn A. Neuronal oscillations in cortical networks. Science 304: 1926-1929, 2004.

Cottereau B, Lorenceau J, Gramfort A, Clerc M, Thirion B, Baillet S. Phase delays within visual cortex shape the response to steady-state visual stimulation. Neuroimage 54: 1919-1929, 2011.

Ermentrout GB, Kleinfeld D. Traveling electrical waves in cortex: insights from phase dynamics and speculation on a computational role. Neuron 29: 33-44, 2001.

Fries P. A mechanism for cognitive dynamics: neuronal communication through neuronal coherence. Trends Cogn Sci 9: 474-480, 2005.

Hari R, Salmelin R. Human cortical oscillations: a neuromagnetic view through the skull. Trends Neurosci 20: 44-49, 1997.

Hindriks R, van Putten MJ, Deco G. Intra-cortical propagation of EEG alpha oscillations. Neuroimage 103: 444-453, 2014.

Hoogenboom N, Schoffelen JM, Oostenveld R, Parkes LM, Fries P. Localizing human visual gamma-band activity in frequency, time and space. Neuroimage 29: 764-773, 2006.

Hughes JR. The phenomenon of travelling waves: a review. Clin Electroencephalogr 26: 1-6, 1995.

Jensen O, Kaiser J, Lachaux JP. Human gamma-frequency oscillations associated with attention and memory. Trends Neurosci 30: 317-324, 2007.

Klimesch W, Hanslmayr S, Sauseng P, Gruber WR, Doppelmayr M. P1 and traveling alpha waves: evidence for evoked oscillations. J Neurophysiol 97: 1311-1318, 2007.

Kösem A, Gramfort A, van Wassenhove V. Encoding of event timing in the phase of neural oscillations. Neuroimage 92: 274-284, 2014.

Lubenov EV, Siapas AG. Hippocampal theta oscillations are travelling waves. Nature 459: 534-539, 2009.

Maris E, Womelsdorf T, Desimone R, Fries P. Rhythmic neuronal synchronization in visual cortex entails spatial phase relation diversity that is modulated by stimulation and attention. Neuroimage 74: 99-116, 2013.

Mitra PP, Pesaran B. Analysis of dynamic brain imaging data. Biophys $J$ 76: 691-708, 1999.

Muller L, Reynaud A, Chavane F, Destexhe A. The stimulus-evoked population response in visual cortex of awake monkey is a propagating wave. Nat Commun 5: 3675, 2014.

Nunez PL. Toward a quantitative description of large-scale neocortical dynamic function and EEG. Behav Brain Sci 23: 371-398, 2000.

Nunez PL, Srinivasan R. Electric Fields of the Brain: the Neurophysics of EEG. Oxford, UK: Oxford Univ. Press, 2005.

Oostenveld R, Fries P, Maris E, Schoffelen JM. FieldTrip: open source software for advanced analysis of MEG, EEG, and invasive electrophysiological data. Comput Intell Neurosci 2011: 156869, 2011.

Percival DB, Walden AT. Spectral Analysis for Physical Applications: Multitaper and Conventional Univariate Techniques. Cambridge, UK: Cambridge Univ. Press, 1993.

van der Meij R, Kahana M, Maris E. Phase-amplitude coupling in human electrocorticography is spatially distributed and phase diverse. J Neurosci 32: 111-123, 2012.

van Pelt S, Boomsma DI, Fries P. Magnetoencephalography in twins reveals a strong genetic determination of the peak frequency of visually induced $\gamma$-band synchronization. J Neurosci 32: 3388-3392, 2012. 